\title{
Computer-assisted therapy for medication-resistant auditory hallucinations: proof-of-concept study
}

\author{
Julian Leff, Geoffrey Williams, Mark A. Huckvale, Maurice Arbuthnot and Alex P. Leff
}

\section{Background}

One in four patients with schizophrenia responds poorly to antipsychotic medication, continuing to hear persecutory auditory hallucinations. Patients who are able to sustain a dialogue with their persecutor feel much more in control.

\section{Aims \\ To develop a computerised system that enables the patient to create an avatar of their persecutor. To encourage them to engage in a dialogue with the avatar, which the therapist is able to control so that the avatar progressively yields control to the patient.}

\section{Method}

Avatar therapy was evaluated by a randomised, single blind, partial crossover trial comparing the novel therapy with treatment as usual (TAU). We used three main outcome measures: (a) the Psychotic Symptom Rating Scale (PSYRATS), hallucinations section; (b) the Omnipotence and Malevolence subscales of the Revised Beliefs About Voices Questionnaire (BAVQ-R); and (C) the Calgary Depression Scale (CDS).

\section{Results}

The control group showed no change over time in their scores on the three assessments, whereas the novel therapy group showed mean reductions in the total PSYRATS score (auditory hallucinations) of $8.75(P=0.003)$ and in the BAVQ-R combined score of omnipotence and malevolence of the voices of $5.88(P=0.004)$. There was no significant reduction in the CDS total score for depression. For the crossover control group, comparison of the period of TAU with the period of avatar therapy confirmed the findings of the previous analysis. The effect size of the therapy was 0.8 .

\section{Conclusions}

Avatar therapy represents a promising treatment for medication-resistant auditory hallucinations. Replication with a larger sample is required before roll-out to clinical settings.

\section{Declaration of interest}

None.
The introduction of antipsychotic medication benefitted many patients and helped to initiate the wave of discharges from psychiatric hospitals. Unfortunately, one in four patients with schizophrenia fails to respond to antipsychotics ${ }^{1}$ and continues to experience persistent auditory hallucinations, which have a major impact on their lives and can lead to suicide. ${ }^{2}$ In an attempt to tackle this problem we developed a novel therapy to give patients control over their 'voices'. The rationale derived from the observation that when people are asked about the worst aspect of hearing voices their invariable response is the helplessness. However, research has shown that patients who can initiate a dialogue with their voice feel much more in control. ${ }^{3}$ Patients are often advised by professionals to ignore the voices and not to engage with them. However, the approach of Romme and colleagues $^{4}$ of encouraging patients to enter into a dialogue with their voices has proved to be therapeutic. Furthermore, the association between trauma of various types in early life and the later development of auditory hallucinations is evidence for an understandable psychological origin for voices, although the exact mechanism has yet to be established. ${ }^{5,6}$ Some patients realise that their low self-esteem, induced by traumatic childhood experiences, is echoed by the voices that harass them. The development of persecutory auditory hallucinations can be formulated as an exteriorisation of a severely critical component of the psyche that cannot be tolerated. ${ }^{7}$ If this is correct, then ignoring the voices negates the possibility of reassimilation of this rejected component of the patient's internal world. In 26 people who heard voices, Chadwick \& Birchwood ${ }^{8}$ studied their experiences and beliefs about the voices. All participants interviewed who heard voices giving them commands, held additional beliefs that if they disobeyed, they would be punished

'See editorial, pp. 394-395, this issue. or even killed. These authors developed a therapy based on asking the patients to test their belief in the dire consequences of disobeying these commands, a strategy that met with some success. An alternative approach of facilitating a dialogue between the patient and their persecutor in which the patient is enabled to gain control and the persecutor mellows, has determined the nature of our novel therapy.

\section{Method}

It is very difficult to establish a dialogue with an invisible entity that repeats the same stereotyped abusive phrases regardless of the patient's response. It was considered that if the persecutor were to be given a human face and made responsive to the patient's speech, a dialogue between the patient and their persecutor could be established with the assistance of a therapist. The therapy is based on computer technology that enables each patient to create an avatar of the entity they believe is talking to them. Construction of an avatar requires a program to create a face, animation software to synchronise lip movements with speech, and software to enable the therapist to speak through the avatar with the voice the patient hears. Commercial software was available for face construction (Facegen Modeller version 3.5.1 for Windows; Singular Inversions, Toronto, Canada; www. facegen.com) and for animation (Annosoft Real-time LipSync SDK 4.0.0.0 for Windows; Annosoft, Richardson, Texas, USA; www.annosoft.com/microphone-lipsinc), but nothing existed to reproduce the voice of the patient's persecutor. Software was developed in-house by M.-H., consisting of programs for building a range of transforms of the therapist's voice, for selection of a voice by the patient, and for the real-time voice transformation. The voice conversion technology was constructed using the approach developed by Stylianou ${ }^{9}$ whereby a probabilistic 
linear transform is applied to the speech spectral envelope in combination with pitch scaling. On average it takes $15 \mathrm{~min}$ for the patient to choose the face (online Fig. DS1), and $25 \mathrm{~min}$ to choose the voice. Patients who did not visualise a face were asked to select a face they would feel comfortable talking to. Patients hearing multiple voices were asked to focus on the dominant voice, or the voice they would most like to be rid of.

During the therapy sessions, the avatar's utterances were produced by the therapist, then voice transformed and played to the patient in another room together with the animated face. The patient's responses were then fed back to the therapist so that the patient effectively interacted with the avatar as an external entity. The therapist could also communicate instructions or advice and encouragement to the patient in their normal voice over a separate audio channel, without involving the avatar. Switching between the two modes of communication was effected via a simple graphical interface. Sound was transferred between the two computers over a standard network connection. The patient was seated in a room and faced a monitor on which their avatar was shown. The therapist sat in an adjacent room and also viewed a screen on which the avatar appeared.

There was an additional voice-changing screen. Clicking on the right side of the screen allowed the therapist to speak to the patient through the avatar using the morphed voice. Clicking on the left side of the screen enabled the therapist to speak to the patient in their normal voice. The patient was prompted by the therapist to enter into a dialogue with their avatar in which the therapist encouraged them to stand up to the avatar. The therapist controlled the avatar so that it progressively came under the patients' control. In addition, over the course of the therapy the character of the avatar was changed by the therapist from being abusive to becoming helpful and supportive of the patient. Our assumption was that patients who are assisted by the therapy to establish control over the avatar would be able to transfer that experience to the persecutory voice. We also considered it possible that the transformation of the avatar from a hostile entity to a well-intentioned supporter might enable the patient to reintegrate the exteriorised component of their psyche. Each session was recorded and transferred to an MP3 (portable digital audio) player for the patients to use outside of the sessions to reinforce their control over their persecutor.

In case participants became very anxious on seeing and hearing the avatar, a prominent red stress button was made available and its purpose explained. Pressing the button caused the avatar to disappear from the monitor, to be replaced by a scene of a tropical beach accompanied by soothing music.

\section{Evaluating the therapy}

\section{Design of the trial}

This was a Phase II proof-of-concept study of the efficacy of a novel therapy. For this purpose the design chosen was a randomised, partial crossover trial, with follow-up of patients who had received the treatment. Patients who were randomised but dropped out of treatment yielded no further data. Choice of the number of sessions was determined by practical considerations: the short duration available to complete the trial (11 months) and the fact that there was only one therapist, J.L. These factors led to a decision to limit the therapy to 6 sessions of $30 \mathrm{~min}$ duration with a 1-week follow-up. An additional follow-up was planned for 3 months after the last therapy session.

Choosing a control treatment for comparison with avatar therapy was problematic and after some deliberation, it was decided that the control group would receive treatment as usual (TAU) for 7 weeks before being offered avatar therapy, although it was recognised that this would not control for the additional attention paid to the patients. Treatment as usual consisted of the patient's ongoing antipsychotic medication prescribed and supervised by their referring psychiatrist.

The power calculation was based on a drop-out rate of $25 \%$ and a reduction in the Omnipotence score (see below) of $35 \%$ with a power of $80 \%$ at $P<0.05$. This degree of reduction was judged to be clinically meaningful. The two inclusion criteria for the study were: hearing persecutory voices for at least 6 months, which had not responded adequately to antipsychotic medication irrespective of diagnosis, and age between 14 and 75. Parental consent was obtained for those under 18. Exclusion criteria were organic brain disease and substance misuse. The study was approved by the West Kent Research Ethics Committee.

\section{Recruitment of patients}

Participants were recruited from the community mental health teams in Camden and Islington Mental Health Trust.

\section{Randomisation procedure}

After baseline assessment, patients were randomised into one of two groups using a computer-generated series with blocks of 12, generated by an independent statistician. The immediate therapy group entered straight into the 7-week block of avatar therapy, whereas the delayed therapy group received TAU for 7 weeks (control block) and were then offered a 7-week block of avatar therapy. This enabled us to look at within- as well as between-group effects. The immediate therapy group did not crossover into a no-therapy block as we expected the therapy to have carry-over effects.

\section{Assessment of patients}

Both groups of patients were assessed at each time point by M.A., a user-researcher who knew neither the group to which each patient was assigned, nor the design of the trial. Interrater reliability between M.A. and J.L. was assessed for 10 patients by Cohen's kappa and ranged from 0.84 to 0.94 . Three questionnaires were administered.

(a) The Psychotic Symptom Rating Scale (PSYRATS) ${ }^{10}$ hallucinations section, which captures information on the frequency and disturbing qualities of the hallucinations.

(b) The revised Beliefs About Voices Questionnaire (BAVQ-R), ${ }^{11}$ which focuses on the patient's beliefs about the 'voices', and thus indexes how likely the voices are to affect behaviour. Two of the five subscales of this instrument were selected a priori for analysis as most pertinent to the predicted effects of the therapy. The Omnipotence scale measures the power of the voice as perceived by the patient, which was expected to reduce since the avatar progressively yields to the patient's assertiveness. The Malevolence scale measures the patient's beliefs about the evil intentions of the voices towards them, which were expected to improve since the avatar is manipulated by the therapist from an initial persecutory role to one supportive of the patient. The scores on these two scales were analysed separately and as a sum total. The key question was whether the changes in the avatar would alter the patients' experience of the voices they hear.

(c) The Calgary Depression Scale (CDS), ${ }^{12}$ since depression is common in schizophrenia. ${ }^{13}$

\section{Data analysis}

Two main analyses were performed: first a comparison of the immediate therapy group with the delayed therapy group to 
establish whether the therapy had an effect on the three main outcome measures. The dependent variable was the change score (absolute difference) between the pre-treatment measure and the post-treatment measure. These were entered into a one-sample $t$-test (null hypothesis: there is no significant effect of the therapy on each outcome measure). All $t$-tests were two-tailed.

The second analysis was within-group, to directly compare the effects of therapy on the delayed therapy group. This was a repeated measures ANOVA on the raw scores at each of the three time points $\left(T_{0}\right.$ : baseline; $T_{1}$ : post 7-week control block; $T_{2}$ : post 7 -week therapy block). If there was a significant effect of time, we then used post hoc $t$-tests to see whether this was over the therapy block $\left(T_{1}\right.$ to $\left.T_{2}\right)$ rather than over the control block $\left(T_{0}-T_{1}\right)$.

We also undertook a third analysis to investigate whether therapy gains were maintained for both groups at the 3-month follow-up. We performed paired $t$-tests comparing the 3-month data with two time points: (a) pre-treatment $v$. follow-up (to see whether patients were still improved compared with baseline); (b) post-treatment $v$. follow-up (to see whether patients continued to improve after avatar therapy).

In addition, we pre-planned a subsidiary analysis of the suicide item on the CDS, rated $0-4$, because of the high risk of suicide in patients with command hallucinations.

We used independent-sample $t$-tests to look for any failure of randomisation in terms of retaining balance between the two groups on the three main outcome variables both at the time of randomisation and at the first follow-up after some patients had dropped out of both groups. The statistical analyses were conducted by A.L.

\section{Results}

Initially, very few referrals for the therapy were made, probably because of the untried nature of the therapy. A steady rate of referrals was only achieved after some dramatic successes with the therapy. Eventually, 27 patients were recruited, all of whom met the inclusion criteria. However, 1 patient refused when approached by the researcher. For demographic data on the 26 included patients see Table 1.

\begin{tabular}{|lc|}
\hline Table 1 Sociodemographic data of patients $(n=26)$ & $n$ \\
\hline Gender & \\
Male & 16 \\
Female & 10 \\
\hline Employment & \\
None & 14 \\
Retired & 3 \\
Student & 6 \\
Part time & 1 \\
Full time & 2 \\
\hline Education & \\
No qualifications & 7 \\
GCSE & 10 \\
A level & 3 \\
Degree & 6 \\
\hline Voice duration, years ${ }^{a}$ & \\
1-5 & 8 \\
6-10 & 3 \\
\hline >10 & 15 \\
\hline Medication compliance & 22 \\
None & 2 \\
Partial & \\
Complete & \\
\hline a. Range 3-30. & 2 \\
\hline
\end{tabular}

The duration of hearing voices for the majority exceeded 10 years. All but two patients were completely or partially adherent with antipsychotic medication. One had stopped the medication 9 months prior to the study because of excessive weight gain and the other participant had stopped 3 years before the study as it had not diminished his hallucinations.

In total 14 patients were randomly assigned to the immediate therapy group and 12 to the delayed therapy group. In our comparison of the immediate therapy group with the delayed therapy group to establish whether the therapy had an effect on the three main outcome measures we found significant positive effects for both the PSYRATS total score $(P=0.003)$, with an average reduction of -8.75 points, and the BAVQ-R total score $(P=0.004)$, with an average reduction of -5.88 points; but we found no significant effect on CDS score $(P=0.423)$, with an average reduction of only -0.94 (Fig. 1).

Our second, within-group, analysis that directly compared the effects of therapy on the delayed therapy group $(n=8)$, confirmed the findings from the first analysis, with significant positive effects for the following measures: (a) PSYRATS total score $(P=0.006)$, with the reduction in score occurring at the expected time point ( $\left.T_{0}-T_{1} P=0.960 ; T_{1}-T_{2} P=0.027\right)$, (b) BAVQ-R score $(P=0.014)$, with the reduction in score again occurring at the expected time point $\left(T_{0}-T_{1} P=0.660, T_{1}-T_{2} P=0.042\right)$ (Fig. 1).

(a)

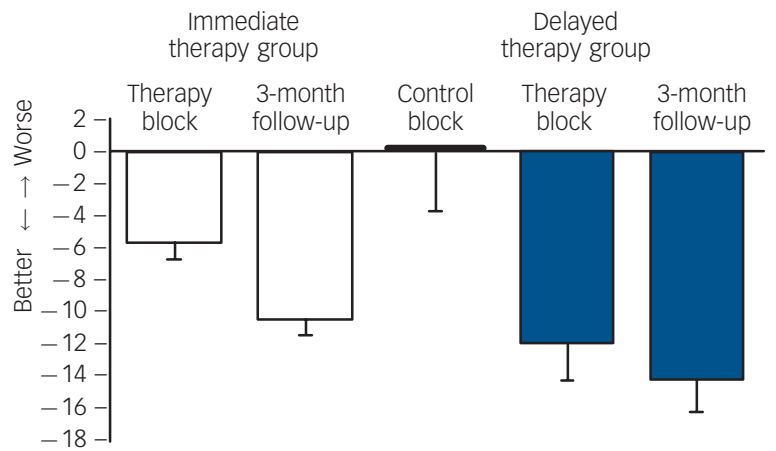

(b)

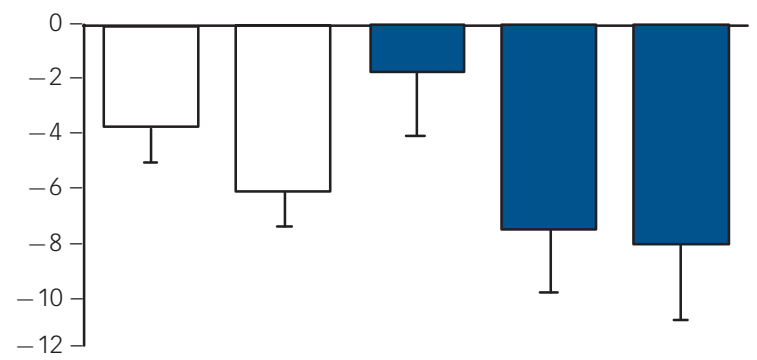

(c)

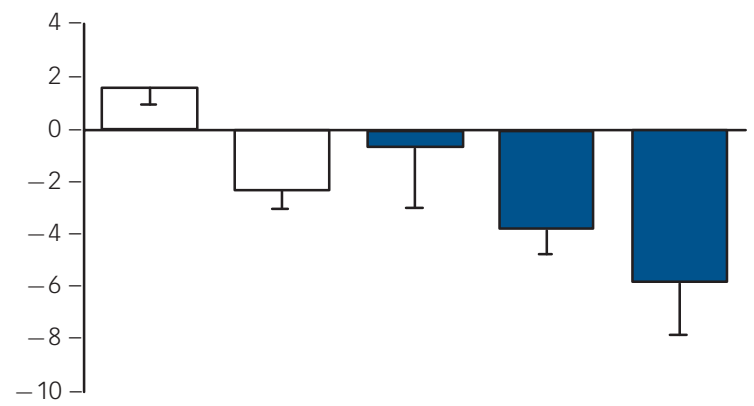

Fig. 1 Effect sizes of avatar therapy on the three main outcome measures for both patient groups.

(a) Psychotic Symptom Rating Scale; (b) revised Beliefs About Voices Questionnaire; (c) Calgary Depression Scale. The immediate therapy group is shown in white and the delayed therapy group in blue. A negative score indicates improvement (post-therapy minus pre-therapy). 
The two outcome measures that reduced significantly across both analyses (PSYRATS and BAVQ-R) are measures of the frequency and quality of the auditory hallucinations, and the delusions patients develop about the voices. The third measure of depressive symptoms was not significantly reduced in any of the comparisons. The effect size of the therapy was 0.8 .

Three months after the therapy ended we successfully followed up the 16 patients who had received the therapy (immediate therapy group $n=8$, delayed therapy group $n=8$ ). We collected full data on 14 patients. One patient lost concentration during the BAVQ-R, which was incomplete, and another patient only consented to complete the PSYRATS. In the third analysis, which investigated whether therapy gains were maintained for both groups at the 3-month follow-up, we found that compared with the pre-treatment scores, the patients remained significantly better on both the PSYRATS $(P<0.001)$, with an average reduction of 12.25 points, and the BAVQ-R total score $(P=0.014)$, with an average reduction of 7.00 points. We also found an effect on the CDS score, $P=0.036$ with an average reduction of 4.13 points. At the post-treatment time point, we found positive changes for both the PSYRATS $(P<0.029)$, with an average reduction of 3.5 points, and the CDS score $(P=0.052)$, with an average reduction of 2.8 points (Fig. 1$)$. This indicates that both the PSYRATS and the CDS continued to improve after the therapy ended. There was no significant effect on the BAVQ-R total score $(P=0.611)$ with an average reduction of only 1.14 points. All three patients whose voices ceased during the therapy were still free of them at the 3-month follow-up. Means and standard deviations for each group at each time point are shown in Table 2.

The average reduction in the BAVQ-R Omnipotence subscale score between the beginning and the end of the therapy was $29.0 \%$ (pre-treatment mean, 12.2; post-treatment mean 8.5 ), close to the reduction of $35 \%$ used for the power calculation. The average reduction in this score between the beginning of the therapy and the 3-month follow-up was 37.9\% (3-month follow-up mean, 7.4).
In our subsidiary analysis of the suicide item on the CDS, we found that by the end of the therapy sessions the score on this item had reduced significantly, $P=0.034$ (mean pre-treatment 0.94; mean post-treatment 0.38 ).

\section{Drop-out rate}

Of the 14 patients in the immediate therapy group, 5 dropped out and one was excluded because of a change in medication. All 12 patients in the delayed therapy group were followed up after 7 weeks and were then offered the therapy. Eight accepted and were followed up again 1 week after the end of the therapy. Hence a total of 16 patients across both groups received the therapy and were followed up. The total drop-out rate was 9 out of 26 $(34.6 \%)$. The refusal of four patients to accept the offer of therapy, and of five patients to complete their course of therapy is mainly accounted for by the fear instilled in patients by their 'voices', which often threaten them if they disobey. ${ }^{10}$ Two patients heard multiple voices and could not concentrate on the avatar because the other voices spoke too loudly at the same time. It took considerable courage for some of the patients to face their avatar. One participant who had been sexually abused by an older man could not bear to see his face, which we deleted, but was able to speak with the avatar, and in fact the voice ceased after 13 years of torment.

In our analysis to test for failure of randomisation in terms of retaining balance between the two groups on the three main outcome variables (Table 3), we found no significant differences between groups both immediately after randomisation (immediate therapy group $n=14$, delayed therapy group $n=12$ ) or after drop out (immediate therapy group $n=8$, delayed therapy group $n=8$ ) (all $P>0.25$ ).

\section{Discussion}

We hypothesised that this novel therapy might achieve some reduction in the frequency and intensity of the auditory

Table 2 Mean values (s.d.) for the main three outcome measures at all time points

\begin{tabular}{|c|c|c|c|c|c|c|c|}
\hline & \multicolumn{3}{|c|}{ Immediate therapy group, mean (s.d.) } & \multicolumn{4}{|c|}{ Delayed therapy group, mean (s.d.) } \\
\hline & Baseline & $\begin{array}{l}\text { Post- } \\
\text { treatment }\end{array}$ & $\begin{array}{l}\text { 3-month } \\
\text { follow-up }\end{array}$ & Baseline & $\begin{array}{l}\text { Pre- } \\
\text { treatment }\end{array}$ & $\begin{array}{l}\text { Post- } \\
\text { treatment }\end{array}$ & $\begin{array}{l}\text { 3-month } \\
\text { follow-up }\end{array}$ \\
\hline Psychotic Symptom Rating Scale & $29.25(4.86)$ & $23.63(8.03)$ & $18.88(8.90)$ & 31.75 (5.39) & $31.88(8.10)$ & $20.00(13.10)$ & $17.75(9.41)$ \\
\hline Revised Beliefs About Voices Questionnaire & $22.63(7.58)$ & $18.88(7.24)$ & $15.57(8.96)$ & $21.38(8.85)$ & $21.00(11.33)$ & $12.37(12.61)$ & $13.43(9.38)$ \\
\hline Calgary Depression Scale & $6.88(4.02)$ & $8.50(5.29)$ & $3.71(2.98)$ & $9.25(2.37)$ & $8.63(8.49)$ & $4.00(1.41)$ & $2.88(3.52)$ \\
\hline
\end{tabular}

Table 3 Comparison of the baseline means (s.d.) for the three main outcome measures between the delayed therapy and the immediate therapy group after randomisation (baseline) and at the first follow-up, after some patients had dropped out of both groups $^{a}$

\begin{tabular}{|c|c|c|c|c|c|c|}
\hline & \multicolumn{3}{|c|}{ Baseline } & \multicolumn{3}{|c|}{ First follow-up } \\
\hline & Mean (s.d.) & $t$ (d.f. $=24)$ & $P$ & Mean (s.d.) & $t$ (d.f. $=14)$ & $P$ \\
\hline Psychotic Symptom Rating Scale & & 0.79 & 0.44 & & 0.97 & 0.35 \\
\hline Delayed therapy group & $33.75(5.55)$ & & & 31.75 (5.39) & & \\
\hline Immediate therapy group & $32.00(5.71)$ & & & $29.25(4.86)$ & & \\
\hline Revised Beliefs About Voices Questionnaire & & -0.69 & 0.50 & & 0.86 & 0.40 \\
\hline Delayed therapy group & $9.17(5.94)$ & & & $9.25(6.69)$ & & \\
\hline Immediate therapy group & $10.93(7.01)$ & & & $6.88(4.02)$ & & \\
\hline Calgary Depression Scale & & -1.15 & 0.26 & & -0.30 & 0.77 \\
\hline Delayed therapy group & $22.67(9.41)$ & & & $21.38(8.85)$ & & \\
\hline Immediate therapy group & $26.50(7.53)$ & & & $22.63(7.58)$ & & \\
\hline
\end{tabular}


hallucinations. In the event, two of the three main outcome measures were significantly reduced immediately after avatar therapy: the PSYRATS and BAVQ-R. They are measures of the frequency and intensity of the auditory hallucinations, the disruption they cause to life, and the beliefs patients develop about their hallucinations, in particular their omnipotence and malevolence. Reductions of this degree are clinically important considering that the patients' hallucinations had failed to respond to many years of the most effective antipsychotic drugs available. The third measure was of depressive symptoms. Although this was not significantly reduced by the end of therapy, by the 3-month follow-up this variable significantly improved from baseline across both the immediate and delayed therapy groups. Depression is common in people with schizophrenia, ${ }^{13}$ and although the therapy did not specifically target depressive symptoms, when patients were rated as having low self-esteem, attempts were made to improve their self-image, with success in some individuals. The significant reduction in the patients' suicidal intent is of considerable clinical import. We investigated whether the individuals who had dropped out had invalidated the randomisation and found that in terms of the three main outcome measures the equivalence of the experimental and control participants had not been affected.

Avatar therapy is very brief, never more than 7 sessions held weekly, each lasting no more than $30 \mathrm{~min}$. In fact, the recordings show that the majority of dialogues with the avatar lasted $15 \mathrm{~min}$ or less. The additional time was occupied with the patient's report on the previous week before the dialogue ensued, and feedback following the session. The outcome that was unexpected was the abrupt cessation of the hallucinations in three of the patients, which remained absent at the 3-month follow-up. In two of the patients their voices stopped after the second session of therapy. One had been hearing the voice of the devil for 16 years and thanked us for giving him his life back. Another participant, for 3.5 years, had been woken every morning at $05.00 \mathrm{~h}$ by a woman's voice that continued throughout the day. He said 'It's as if she left the room'.

\section{How did the therapy work?}

There are several possible explanations for the efficacy of avatar therapy. The therapist takes the patients' experiences at face value and assists them to actualise their persecutor, validating the patient's experience. ${ }^{9}$ Although patients interact with the avatar as though it is a real person, because it is their creation they know that it cannot harm them, as opposed to the voices, which they fear. They can take risks with the avatar, standing up to it and telling it forcefully to leave them alone, behaviour they would not attempt with their delusional persecutor. Once they gain the courage to confront the avatar, they learn to do the same with their persecutor, as evidenced by the significant reduction in the perceived power of their persecutor. During the course of therapy the avatar changes its nature, ceasing to abuse the patient after one or two sessions, and becoming friendly and supportive. The MP3, which is given to each patient to keep, contains all their recorded sessions. Both the therapist and the avatar encourage the patients to listen to their MP3 when harassed by the voices. We describe it to them as 'a therapist in their pocket'. It is possible that the continued improvement after the end of the therapy was attributable to the use of the MP3. However, we did not quantify the patients' use of the MP3 during this period, so we can only speculate about this.

Many of the patients in our study had very low self-esteem. The link made by the therapist and the avatar between the patients' low self-esteem and the abuse from the voices, helped some patients to recognise that the voices originate within their own mind, and could ameliorate if the patient began to recognise their own good qualities.

\section{Limitations of the study}

By comparing avatar therapy with TAU we did not control for the time and attention paid by the therapist to patients receiving the therapy. In the proposed replication study we will include an active control of supportive counselling. We will also test the adequacy of the masking of the assessor. We did not conduct an intention-to-treat analysis since this is inappropriate for a Phase II trial of efficacy of a new treatment of unknown effect: 'Phase II trials decide whether the new treatment is promising and warrants further investigation in a large-scale randomised Phase III clinical trial based on an observed response rate that appears to be an improvement over the standard treatment or other experimental treatments. ${ }^{14}$

We have learnt that avatar therapy is not suitable for all patients, but this proof-of-concept study provides evidence that it is effective for those who can tolerate it. The high drop-out rate is a matter of concern and the acceptability of avatar therapy will be addressed in more detail by the Phase III replication trial, which is already funded.

For practical reasons only a single therapist delivered the therapy in the reported study. This raises the questions of whether the skills required to achieve these positive results can be taught to others, and the length of training necessary. In preparation for the independent replication trial in London, a technical manual and a clinical manual have been completed.

\footnotetext{
Julian Leff, FRCPsych, Department of Mental Health Sciences, Royal Free and University College Medical School, London; Geoffrey Williams, PhD, Mark A. Huckvale, PhD, Department of Speech, Hearing and Phonetic Sciences, University College London; Maurice Arbuthnot, MA, Department of Mental Health Sciences, Royal Free and University College Medical School, London; Alex P. Leff, PhD, MRCP, Institute of Cognitive Neuroscience and

Department of Brain, Repair and Rehabilitation, Institute of Neurology, University College London, UK.

Correspondence Julian Leff, c/O The Royal College of Psychiatrists,

17 Belgrave Square, London SW1X 8PG, UK. Email:.j.leff@ucl.ac.uk

First received 24 Apr 2012, final revision 11 Dec 2012, accepted 12 Dec 2012
}

\section{Funding}

The study was funded by the National Institute of Health Research (RC-PG-0308-10232) and Bridging Funding from Camden \& Islington NHS Foundation Trust.

\section{References}

1 Kane JM. Treatment resistant schizophrenic patients. J Clin Psychol 2007; 57 (suppl 9): 35-40.

2 van Os J, Kapur S. Schizophrenia. Lancet 2009; 374: 635-45.

3 Nayani THS, David A. The auditory hallucination: a phenomenological survey. Psychol Med 1996; 26: 177-89.

4 Romme M, Escher S, Dillon J, Corstens D, Morris M. Living with Voices: 50 Stories of Recovery. PCCS Books, 2009.

5 Read J, Fink P, Rudegaier T, Felitti V, Whitfield C. Child maltreatment and psychosis: a return to a genuinely integrated bio-psychosocial model. Clin Schizophr Relat Psychoses 2008; 2: 235-54.

6 Bebbington $\mathrm{P}$, Jonas $\mathrm{S}$, Kuipers $\mathrm{E}$, King $\mathrm{M}$, Cooper $\mathrm{C}$, Brugha $\mathrm{T}$, et al. Childhood sexual abuse and psychosis: data from a cross-sectional national psychiatric survey in England. Br J Psychiatry 2011; 199: 29-37.

7 Corstens D, Longden D, May R. Talking with voices: exploring what is expressed by the voices people hear. Psychosis 2012; 4: 95-104.

8 Chadwick $\mathrm{P}$, Birchwood $\mathrm{M}$. The omnipotence of voices, a cognitive approach to auditory hallucinations. Br J Psychiatry 1994; 164: 190-201.

9 Stylianou Y. Continuous probabilistic transform for voice conversion. IEEE Trans Speech and Audio Proc 1998; 6: 131-42. 
10 Haddock G, McCarron J, Tarrier N, Farragher EB. Scales to measure dimensions of hallucinations and delusions: the psychotic symptom rating scales. Psychol Med 1999; 29: 879-98.

11 Chadwick $P$, Lees S, Birchwood M. The revised Beliefs About Voices Questionnaire (BAVQ-R). Br J Psychiatry 2000; 177: 229-32.

12 Addington $\mathrm{D}$, Addington J, Maticka-Tyndale E. Assessing depression in schizophrenia: the Calgary Depression Scale. Br J Psychiatry 1993; 163 (suppl 22): $539-44$
13 Leff J, Tress K, Edwards B. The clinical course of depressive symptoms in schizophrenia. Schizophr Res 1988; 1: 25-30.

14 Seymour L, Ivy SP, Sargent D, Baker L, Rubinstein L, Retain MJ. The design of Phase II clinical trials testing cancer therapeutics: consensus recommendations from the clinical design task force of the national cancer institute investigational drug steering committee. Clin Cancer Res 2010; 16: 1764-9.

\section{German E. Berrios}

Karl Jaspers has the dubious distinction of being more often quoted than read. John Hoenig, one of the translators of Allgemeine Psychopathologie into English, tells that he once saw the book in the office of a famous US psychiatrist and 'asked him whether he liked it. He replied half jokingly, nobody reads it, but it is obligatory to have it seen on your shelf'

Allgemeine Psychopathologie has been sacralised as the fons et origo of all psychopathology. Very few bother to distinguish between the small first edition (1913, 332 pages) and the very large 7th edition (1959, 748 pages), the one rendered into English, Spanish, and Italian. (The French version is a translation of the 3rd edition, 1922.) The sad part of this is that Jaspers is not idolised on account of his main contributions, which are: a) the articulation of a moderately (Kantian) constructionist view of mental symptoms and disorders, and b) scepticism against their full naturalisation. Instead, Allgemeine Psychopathologie is cherry-picked on his views on delusions, understanding, morbid jealousy, pseudohallucinations, process, personality, etc., etc., most of which, in fact, were already available in the literature since before his time.

Allgemeine Psychopathologie cannot be understood without knowledge of its historical background. When the book first appeared in 1913, there were already in circulation substantial works on 'psychopathology': Allgemeine Psychopathologie (Emminghaus, 1878) - which provided the name for Jaspers's book; Manuale di Semejotica delle Malattie Mentali (Morselli, 2 Vols. 1885; 1894); Vorlesungen über Psychopathologie (Störring, 1900); Éléments de Sémiologie et Clinique Mentales (Chaslin, 1912); Traité International de Psychologie Pathologique (4 vols) (Marie, 1910-1912), etc. Although the term 'Psycho-pathologie' had been introduced by Feuchtersleben in 1845, the discipline it came to name took the best part of a century to be put together. Moulded on French medical séméiotique, descriptive psychopathology was meant to configure (or capture) the phenomena of madness and other mental afflictions and soon became the official language of psychiatry.

Representing a complete synthesis of the psychopathological knowledge available at the time, the works mentioned above were available at the Library of the Department of Psychiatry in Heidelberg and offered the young Jaspers a model on which to base his own work. It is not widely known that Allgemeine Psychopathologie was not meant to be a book on 'psychopathology'. In the autumn of 1910, Ferdinand Springer invited Wilmanns (then Head of Department at Heidelberg) to write an 'Introduction to Psychiatry.'. A forensic psychiatrist, Wilmanns turned down the invitation and this was eventually passed on to the young Jaspers. 'Seized by enthusiasm', Jaspers saw his task as bringing 'order at least into the factual data'. He did just that: the first edition of Allgemeine Psychopathologie is therefore a cento of clinical information (well known at the time) linked together by Jaspers' own version of Kantian constructionism and Diltheyian ideas on understanding and 'descriptive psychology'. Of Husserl there is far less, in spite of what is claimed by some Jaspersian exegetists. The book fell almost flat. Journal reviews saw it as yet another introduction to psychiatry and some criticised its theoriticism.

In 2013, it is not a bad idea to ask what else of value would Jaspers have bequeathed to psychiatry had he completed his training and stayed in the profession. Alas, like in the case of Störring, Ziehen and other great men who left psychiatry for philosophy, we shall never know. Be that as it may, there is no better homage to a writer than actually reading his work. Allgemeine Psychopathologie does need deeper analysis not starry-eyed contemplation. All seven editions must be collated so that the non-Jaspersian contributions to the latter ones may be identified.

When this analysis is completed, I suspect, Allgemeine Psychopathologie will still be considered as important but its value will reside neither in its 'clinical content' nor in its 'phenomenology'. It will endure because it demonstrates that descriptive psychopathology is not an 'objective and eternal' algorithm built to capture 'clinical' facts but instead it is a metalanguage through which culture is able to govern the presentations and experiences of madness. Let 2013 be the year when this new approach to Jaspers' work truly begins. 